\title{
Teste de Complementação de Letras: Estímulos para uso no Brasil
}

\author{
Sabine Pompéia \\ Universidade Federal de São Paulo \\ Ângela Tavares Paes \\ Instituto Dante Pazzanese - São Paulo \\ Orlando Francisco Amodeo Bueno \\ Universidade Federal de São Paulo
}

\begin{abstract}
RESUMO - Testes de complementação de letras (em inglês "stem-completion tests") são amplamente utilizado na literatura internacional para avaliar memória. Não existem no Brasil, porém, trabalhos que propõem estímulos adequados para uso nesse tipo de teste. O objetivo do presente experimento foi selecionar conjuntos de três letras (tríades) que completam palavras em português do Brasil adequadas para maximizar a verificação de memória. Foram obtidos padrões de complementação ao acaso de 137 tríades que completam pelo menos 10 outras palavras comuns em nosso idioma e manipulados fatores que poderiam influenciar nas estimativas de memória usando essas tríades. Verificou-se que, dependendo da probabilidade de complementação das tríades com a palavra mais freqüentemente utilizada ao acaso, não é possível verificar efeitos de memória; ou seja, a taxa de complementação de estímulos familiares (previamente expostos) não é distinguível da linha de base. Foram propostas diretrizes para a seleção de tríades e estímulos adequados para maximizar a obtenção de índices de memória em testes indiretos de complementação.
\end{abstract}

Palavras-chave: memória; complementação; testes indiretos; testes diretos.

\section{Word-Stem Completion: Stimuli for Use in Brazil}

\begin{abstract}
Stem-completion tests are commonly used to assess memory. In Brazil, however, there are no publications that propose adequate stimuli for use in this type of task. Our objective was to select adequate word-stems to maximize memory assessment using this task in Brazil. We obtained chance-completion profiles of 137 word-stems that completed at least 10 common words in Portuguese from Brazil and manipulated factors that could influence memory estimates using these stems. We found that depending on the chance-completion of the most frequently used word to complete a stem it is not possible to show memory effects; that is, completion with familiar stimuli (seen before) is not distinguishable from baseline. Guidelines are proposed for the selection of adequate stems and stimuli to maximize the acquisition of memory estimates using indirect stem-completion tasks.
\end{abstract}

Key words: memory, stem-completion, direct tests, indirect tests.

Testes de complementação de conjuntos iniciais de letras para formar palavras (em inglês "stem-completion tests") têm sido extensivamente utilizados para avaliar uma ampla gama de fatores que influenciam a memória de longo prazo (Graf \& Williams, 1987). Em geral esses "stems" são compostos de conjuntos de três letras (aqui denominados de tríades), como $\mathrm{ABO}$ que pode completar palavras como ABÓBORA. Esse tipo de teste não exige equipamentos sofisticados, envolve baixos custos e, sobretudo, é de fácil realização, o que permite que seja utilizado para avaliar desde pacientes com deficiências psicomotoras e cognitivas até voluntários com alto grau de escolaridade.

1 Apoio Financeiro: FAPESP e AFIP. Agradecimentos: à Dra. Elizabeth Kira, Dr. Dalton F. de Andrade, Fábio T. Montesano e Leandra Fernandes do "Centro de Estatística Aplicada (CEA) da Faculdade de Matemática e Estatística da USP", que auxiliaram na criação do desenho experimental; à Ruth Ferreira dos Santos Galduróz, Ricardo Tabach e Cristiane Chacur, pela ajuda na coleta de dados.

2 Endereço: R. Napoleão de Barros, 925. CEP: 04024-002. São Paulo - SP. Tel: 11.55390155. E-mail: ofabueno@ psicobio.epm.br
Para aplicação do teste de complementação de tríades, é inicialmente necessária uma fase de apresentação dos estímulos que serão utilizados para avaliar a memória (fase de apresentação, codificação, aprendizado, ou fase de estudo). Subseqüientemente, em outra fase (fase teste ou fase de evocação), solicita-se que sejam completadas tríades iniciais de palavras que correspondem às palavras vistas na fase de apresentação. A memória é verificada quando a probabilidade estimada de complementação de uma tríade com uma palavra após essa ter sido apresentada na primeira fase (palavras familiares, ou pré-ativadas) é maior que a probabilidade de complementação com essa mesma palavra ao acaso, isto é, a probabilidade de que essa mesma palavra seja utilizada para completar a tríade sem que o sujeito tenha sido a ela apresentado.

É complexa a determinação do tipo de memória avaliada em cada tipo de teste. Durante anos equiparou-se "testes de memória" com os "tipos de memória" que supostamente seriam por eles avaliados (Richardson-Klavehn \& Bjork, 1988). A memória explícita, por exemplo, tendia a ser considerada aquela observada em tarefas de memória explícita, criando uma espécie de caracterização circular da memória de 
longo prazo (Richardson-Klavehn \& Bjork, 1988). No final da década de 80 , porém, a instrução dada na fase de teste passou a ser considerada de grande importância na distinção dos subtipos de memória de longo prazo avaliados. Johnson e Hasher (1987) propuseram que os testes de memória fossem subdivididos em diretos e indiretos, de acordo com suas instruções. Testes diretos envolvem instruções que solicitam que os sujeitos recordem informações de um episódio de aprendizado, mencionando o tipo, contexto ou tempo em que o evento ocorreu, como é verificado em testes de reconhecimento, que requerem que os sujeitos apontem estímulos aos quais foram apresentados anteriormente (Richardson-Klavehn \& Bjork, 1988). Neste caso, a "intenção" de recordar informações aprendidas anteriormente supostamente levaria, pelo menos preponderantemente, à utilização de estratégias explícitas de recordação. Testes indiretos, por sua vez, envolvem atividades motora ou cognitiva relacionadas a um episódio de aprendizado sem que as instruções se refiram a esses eventos. Isso permite minimizar a recordação "intencional" de informações adquiridas anteriormente, o que caracterizaria recordação preponderante de memória implícita.

Testes como os de complementação de tríades podem envolver tanto instruções diretas como indiretas, permitindo que sejam utilizados para avaliar subtipos distintos de memória de longo prazo. A instrução pode ser "complete a tríade com uma palavra que viu" (teste direto), instrução que, a princípio, desencadeia uso preponderante de memória explícita/episódica, ou "complete com a primeira palavra que vem à mente" (teste indireto), a instrução clássica para avaliar a pré-ativação ("repetition-priming"), um subtipo de memória implícita de longo prazo (Graf \& Schacter, 1985; Magila \& Xavier, 1999). Todavia, deve-se ter cuidado ao considerar que o desempenho em testes diretos equivale ao uso de memória explícita e o de testes indiretos, ao de memória implícita, pois diversos autores apontam que nenhum teste é capaz de mensurar o uso de um só tipo de memória (ver Richardson-Klavehn \& Bjork, 1988; Toth, Lindsay \& Jacoby, 1992; Jacoby, Toth \& Yonelinas, 1993).

Sabe-se que a frequiência de uso na língua e o comprimento de palavras constituem as mais importantes variáveis na determinação das palavras utilizadas como complementações (Graf e Williams, 1987): palavras mais freqüentes e mais curtas tendem a ser completadas mais freqüentemente que as menos freqüentes e mais longas. Contudo, o perfil de complementação ao acaso das 40 tríades estudadas por Graf e Williams (1987) mostra que mais da metade não foi completada em primeiro lugar com a palavra mais curta ou mais freqüente na língua inglesa. Portanto, é provável que, além desses, outros fatores desconhecidos, ou uma combinação de fatores, estejam em jogo na determinação de quais palavras são mais utilizadas para completar tríades. Como nenhuma variável parece ser realmente preditora das palavras geradas a partir de uma tríade, é preciso determinar empiricamente quais palavras (e com qual probabilidade) são utilizadas para completar tríades em cada idioma no qual se pretende empregar testes de complementação, como é o caso do trabalho de Graf e Williams (1987) para a língua inglesa. Não existem, porém, trabalhos que estudaram esses padrões de complementação no Brasil.

Padrões de complementação devem ser determinados para tríades que completam diversas palavras, pois é critério con- sensual na literatura que as palavras empregadas em testes como os de complementação de tríades devem compartilhar suas tríades com diversas outras palavras comuns (Graf \& Williams, 1987). Note, por exemplo, que a tríade BEX só pode ser completada com a palavra BEXIGA (ou palavras derivadas desta). Assim, tendo em vista que testes de complementação são muito fáceis, tanto sujeitos que viram como os que não viram essa palavra na fase de apresentação têm que utilizar essa palavra como complementação. Isso impossibilita que se determine se para tal complementação o sujeito usou ou não memória da fase de apresentação, a não ser que se mostre que o tempo de complementação de sujeitos que viram a palavra na fase anterior é menor do que o dos que não a viram. A mensuração de tempo de complementação, porém, é complexa e exige equipamentos sofisticados.

Como já mencionado, a constatação de uso de memória é feita pela subtração de complementação ao acaso da probabilidade de complementação com palavras familiares. $\mathrm{O}$ controle ideal para esse tipo de teste envolve o emprego de pelo menos duas listas de estímulos equiparadas em termos da probabilidade média de complementação; cada sujeito deve completar tríades com palavras familiares (de uma lista) e outras com palavras não vistas (de outra lista), familiares a outros sujeitos da mesma amostra, do mesmo experimento. Para tal, não basta selecionar tríades/palavras aleatoriamente para construir um teste de complementação. É preciso conhecer as probabilidades de complementação de cada palavra utilizada, o que só é possível se o perfil de complementação for estabelecido no idioma em que se realizará o teste.

Considerando a necessidade de se conhecer quais são as palavras geradas a partir de tríades em português do Brasil e a frequiência ou probabilidade de aparecimento das mesmas, procuramos obter, na primeira fase deste trabalho (experimento 1), um perfil de complementação ao acaso de tríades que podem ser completadas com pelo menos 10 palavras comuns em nosso idioma, critério este amplamente utilizado para selecionar tríades para avaliar a memória (Graf \& Williams, 1987).

\section{Experimento 1}

\section{Sujeitos}

\section{Método}

Cem estudantes de $2^{\circ}$ grau (27 homens) com idade média de 20 anos ( $\mathrm{DP}=5,3)$ para os quais o português era a primeira língua.

\section{Material}

Foram selecionados todos os conjuntos de três letras (tríades) que completam pelo menos 10 palavras comuns através de um dicionário da língua portuguesa de ampla aceitação (Ferreira, 1993). Variações como "chapéu", "chapeleira", "chapelaria", etc., foram computadas como uma mesma palavra.

\section{Procedimento}

Esse experimento fez parte de um estudo amplo que foi aprovado em 12/04/1996 pela Comissão de Ética Médica 
da Universidade Federal de São Paulo. Ele foi realizado durante as aulas após os sujeitos terem assinado termos de consentimento informado. Formulários listando as 137 tríades selecionadas foram apresentados aos sujeitos. Cada sujeito deveria completar cada tríade com duas palavras de modo a minimizar a perda de dados caso houvesse erros na complementação na primeira tentativa. Os sujeitos foram instruídos a completar as tríades "com as primeiras palavras que lhes viessem à mente", evitando usar nomes próprios e palavras no plural.

A computação dos dados foi realizada da seguinte forma:

1. A primeira complementação de cada tríade foi utilizada quando a palavra foi corretamente grafada ou incluia erros de ortografia que não envolvessem as 3 primeiras letras (Ex.: embrema ao invés de emblema; repolso ao invés de repouso). Palavras de diferentes gêneros e tempos verbais foram consideradas independentes, enquanto que aquelas no plural foram somadas às no singular, a não ser que correspondessem a coisas distintas (Ex.: palma e palmas).

2. Utilizou-se a segunda alternativa de complementação quando a primeira era ilegível, não ocorria no dicionário, ou quando: a) havia erro de ortografia nas três primeiras letras (Ex.: senário ao invés de cenário; bisouro ao invés de besouro; indioma ao invés de idioma); b) ocorria complementação com nomes próprios de pessoas (Ex.: Maria, Marcos), mas não de substantivos que poderiam ser um nome ou não, como a palavra "marco"; c) era palavra de três letras no plural (Ex.: cores, aves); d) era palavra que trocava a letra "c" da tríade por "ç" na complementação (Ex.: maça para completar a tríade MAC; açoite para ACO); e) a palavra era um substantivo composto (Ex.: para-quedas).

\section{Resultados e Discussão}

Devido à grande quantidade de dados, não é possível arrolar aqui todas as complementações das 137 tríades. A lista completa pode ser encontrados no site www.unifesp.br/ dpsicobio/adap/adapta.htm.

Determinadas palavras foram usadas com frequiência bastante elevada para completar as respectivas tríades iniciais. A título ilustrativo, para a tríade CIR a palavra mais completada foi CIRCO, com $75 \%$ de complementações. As demais palavras utilizadas foram: círculo (13\%), cirurgia $(4 \%)$, circular $(3 \%)$, ciranda $(2 \%)$, circuito $(1 \%)$, circunstância (1\%) e cirurgião (1\%).

Houve grande variabilidade na probabilidade de complementação das palavra mais utilizadas para completar cada tríade. Por exemplo, a palavra mais utilizada para completar a tríade CON foi CONCURSO, utilizada por 5\% dos sujeitos; já para a tríade CAR, a palavra mais utilizada foi CARRO, completada por $73 \%$ dos voluntários. Essa grande variabilidade motivou a realização de um segundo experimento pela seguinte razão: postulou-se que essas probabilidades variáveis poderiam levar a distorções na estimativa de memória através do uso desse tipo de teste, pois a palavra mais utilizada ao acaso para completar uma tríade é que "compete" como complementação com a palavra utilizada para avaliar a memória. Por exemplo, se for considerado que para a tríade CIR a memória para estímulos que exibem baixa probabilidade de serem completados ao acaso, como a palavra CIRURGIÃO (1\%), não sobrepuja a tendência de utilizar palavras de alta probabilidade como complementação (ex. CIRCO, 75\%), não seria possível avaliar a memória para CIRURGIÃO porque a tríade CIR seria tendencialmente completada com CIRCO, mesmo que essa palavra não tivesse sido apresentada antes. Diferentemente, se a memória para uma palavra com alta probabilidade estimada de complementação interage positivamente à sua já elevada probabilidade de complementação ao acaso, a estimativa de memória para esses estímulos poderia ser exageradamente maior que para outros estímulos com menor probabilidade estimada de complementação. Exemplificando, a apresentação de CIRCO poderia elevar sua complementação em $20 \%$ enquanto que a apresentação de CIRURGIÃO, somente em $5 \%$. Ademais, palavras que completam uma tríade com freqüência muito alta poderiam impossibilitar a verificação de memória, pois sua complementação após a exposição poderia não ser distinguível do desempenho ao acaso de complementação das mesmas, que já é elevado (efeito teto). Nesse caso, mesmo que a palavra CIRCO fosse apresentada antes do teste, não seria possível mostrar um efeito de memória para ela porque a probabilidade estimada de complementação não se diferenciaria de $75 \%$. Diferentemente, é provável que o padrão de complementações para tríades como CON, que não apresentam palavras completadas ao acaso com mais que 5\% de probabilidade, levem a estimativas de memória bastante diferentes; nesse caso não haveria palavras específicas que poderiam "competir" com a palavra memorizada caso essa não fosse a complementação ao acaso mais provável.

O objetivo do segundo experimento foi, portanto, determinar de que modo a palavra mais utilizada para completar sua tríade influencia na estimativa de memória para estímulos com baixa e alta probabilidade de serem completados ao acaso utilizando testes indiretos de complementação de tríades, de modo a selecionar as melhores tríades e estímulos a serem utilizados em testes de complementação no Brasil.

Optou-se por realizar o teste de complementação de tríades com instrução indireta no experimento 2 . O motivo para essa escolha foi que essa é a instrução clássica empregada na literatura e, portanto, seria importante que esse experimento refletisse o tipo de teste mais usado internacionalmente. Além disso, tomou-se o cuidado de diminuir a possibilidade do emprego de estratégias explícitas durante a realização do teste indireto de complementação através da combinação de codificação perceptual e realização de tarefa de interferência entre as fases de apresentação e teste (ver Richardson-Klavehn \& Bjork, 1988; Toth, 2000).

\section{Experimento 2}

\section{Sujeitos}

\section{Método}

Noventa estudantes universitários ( 24 homens) com idade média de 22,3 anos ( $\mathrm{DP}=3,24$ ) para os quais o português era a primeira língua. Eles foram divididos em 3 grupos de 30 indivíduos. 


\section{Material}

Mais de $90 \%$ das palavras mais freqüentemente utilizadas para completar cada tríade do experimento 1 (denominadas aqui de "palavras de alta probabilidade estimada de complementação"; APC) foram completadas com probabilidade entre 5 e $55 \%$. Essas tríades foram classificadas em faixas segundo as porcentagens estimadas das suas complementações mais frequientes (ou palavras com APC) (5-15, 16-25, 26-35, $36-45$, 46-55\% de complementações). Essas faixas foram selecionadas de modo a contemplar um número aproximado de tríades por faixa. Selecionou-se, para cada uma das 5 faixas, seis tríades que tinham um número de palavras alternativas de complementação similar e que exibiam palavras com APC com probabilidade o mais próxima possível dos valores centrais da faixa em questão (por exemplo, para faixa 5-15, as palavras com APC deveriam corresponder a valores próximos a 10\%). Essas tríades foram então numeradas de itens 1 a 6 de acordo com a porcentagem de complementação, de menor a maior (ver tabela 1 ).
Para cada uma das tríades foram selecionadas duas palavras, a com APC (que variava de acordo com as faixas) e uma com baixa probabilidade estimada de complementação (BPC), fixada a priori como entre 4-7\% (ver tabela 1). As probabilidades das palavras com BPC foram fixadas porque a utilização de palavras com probabilidade estimada de complementação ao acaso menores que $4 \%$ praticamente inviabilizaria a determinação da complementação ao acaso com uma amostra menor que 90 indivíduos. Houve cinco tríades que exibiram palavras de APC maiores que 55\% (ABO, BIS, CAR, CIR e MAC), número pequeno demais para possibilitar a inclusão de outra faixa no presente experimento

Construção de listas:

1. Listas de apresentação de palavras (ver tabela 2): três listas foram construídas (uma apresentada a cada grupo) incluindo: a) vinte estímulos, dez com APC e dez com BPC com tríades distintas (somente a complementação com esses estímulos foi utilizada na análise); b) quatro palavras que consistiam de exemplos dados na explicação da primeira tarefa, logo no início para que os sujeitos se acostumassem

Tabela 1. Tríades selecionadas por faixa de probabilidade de complementação, número de palavras alternativas utilizadas como complementações (ALT.) para a tríade e palavras de alta (APC) e baixa (BPC) probabilidade de complementação, precedidas de suas probabilidades (prob.) de complementação e seguidas, entre parênteses, de sua freqüência* de ocorrência em português escrito do Brasil por milhão de palavras.

\begin{tabular}{|c|c|c|c|c|c|c|c|}
\hline \multirow[b]{2}{*}{ Faixa } & \multirow[b]{2}{*}{ Item } & \multirow[b]{2}{*}{ Tríade } & \multirow[b]{2}{*}{ Alt. } & \multicolumn{2}{|c|}{ Alta Probabilidade } & \multicolumn{2}{|c|}{ Baixa Probabilidade } \\
\hline & & & & prob. $(\%)$ & $\begin{array}{c}\text { palavra } \\
\text { (freq. língua*) }\end{array}$ & prob. $(\%)$ & $\begin{array}{c}\text { palavra } \\
\text { (freq. língua*) }\end{array}$ \\
\hline \multirow{6}{*}{$1(5-15 \%)$} & 1 & GRA & 32 & 10 & grade $(<1)$ & 5 & grampo $(<1)$ \\
\hline & 2 & REV & 30 & 10 & revender (1) & 5 & revelar (20) \\
\hline & 3 & INV & 36 & 11 & inventar (6) & 5 & invenção (12) \\
\hline & 4 & $\mathrm{APO}$ & 26 & 13 & apoio (215) & 5 & aposta (39) \\
\hline & 5 & TRI & 29 & 13 & trigo (17) & 5 & trilho (1) \\
\hline & 6 & CAT & 26 & 14 & catar $(<1)$ & 4 & cativar $(<1)$ \\
\hline \multirow{6}{*}{$2(16-25 \%)$} & 1 & PAL & 22 & 18 & palito (1) & 5 & palha (5) \\
\hline & 2 & BAL & 19 & 19 & balde (2) & 4 & baleia (4) \\
\hline & 3 & EXP & 23 & 19 & exposição (122) & 5 & explicar (46) \\
\hline & 4 & MEN & 20 & 19 & mente (18) & 4 & menta $(<1)$ \\
\hline & 5 & ASS & 25 & 22 & assinatura (38) & 5 & assistente (23) \\
\hline & 6 & ANT & 24 & 23 & anta $(<1)$ & 5 & antes (491) \\
\hline \multirow{6}{*}{$3(26-35 \%)$} & 1 & ANA & 24 & 28 & analfabeto (2) & 7 & analisar (31) \\
\hline & 2 & BAI & 18 & 29 & baiano (42) & 4 & bailar $(<1)$ \\
\hline & 3 & FER & 29 & 29 & ferramenta (6) & 6 & ferrugem (2) \\
\hline & 4 & POR & 16 & 31 & porco (7) & 5 & porque (599) \\
\hline & 5 & CRI & 22 & 32 & criança (116) & 5 & crítico (48) \\
\hline & 6 & IND & 32 & 33 & indústria (220) & 5 & índio (35) \\
\hline \multirow{6}{*}{$4(36-45 \%)$} & 1 & TRE & 23 & 37 & trem (36) & 5 & treino (57) \\
\hline & 2 & CAM & 20 & 38 & cama (26) & 4 & camisa (37) \\
\hline & 3 & CAS & 25 & 38 & casa (597) & 5 & castelo (24) \\
\hline & 4 & SER & 25 & 38 & serra (126) & 5 & serrote $(<1)$ \\
\hline & 5 & PEN & 23 & 39 & pente (2) & 4 & peneira (3) \\
\hline & 6 & MER & 19 & 39 & mercado (656) & 6 & mercadoria (11) \\
\hline \multirow{6}{*}{$5(46-55 \%)$} & 1 & BAR & 23 & 48 & barco (25) & 5 & barba (7) \\
\hline & 2 & REF & 15 & 49 & referência (48) & 5 & refeição (14) \\
\hline & 3 & GAL & 15 & 50 & galinha (8) & 5 & galeria (34) \\
\hline & 4 & $\mathrm{ACE}$ & 21 & 51 & acerola $(<1)$ & 5 & aceitar (40) \\
\hline & 5 & AVE & 14 & 52 & avestruz (2) & 6 & avenida (94) \\
\hline & 6 & $\mathrm{AMO}$ & 19 & 54 & amor (104) & 7 & amoroso (13) \\
\hline
\end{tabular}

Nota: * freqüência obtida de www.corpus.f2s.com. 
Tabela 2. Estímulos de alta (APC) e baixa (BPC) probabilidades de complementação ao acaso empregados no estudo, a faixa de probabilidade de complementação às quais pertencem e as tríades utilizadas no teste de recordação/complementação em cada um dos 3 grupos.

\begin{tabular}{|c|c|c|c|c|c|}
\hline \multirow[b]{2}{*}{ Grupo } & \multirow[b]{2}{*}{ Faixa } & \multicolumn{2}{|c|}{ Apresentação } & \multicolumn{2}{|c|}{ Recordação/complementação } \\
\hline & & $\begin{array}{c}\text { Alta probabilidade } \\
\text { (APC) }\end{array}$ & $\begin{array}{c}\text { Baixa probabilidade } \\
\text { (BPC) }\end{array}$ & Tríades familiares & $\begin{array}{c}\text { Tríades não fami- } \\
\text { liares }\end{array}$ \\
\hline \multirow{10}{*}{1} & 1 & grade & invenção & GRA INV & TRI \\
\hline & & revender & aposta & REV APO & CAT \\
\hline & 2 & exposição & assistente & EXP ASS & PAL \\
\hline & & mente & antes & MEN ANT & BAL \\
\hline & 3 & criança & analisar & CRI ANA & FER \\
\hline & & indústria & bailar & IND BAI & POR \\
\hline & 4 & trem & castelo & TRE CAS & PEN \\
\hline & & cama & serrote & CAM SER & MER \\
\hline & 5 & galinha & avenida & GAL AVE & BAR \\
\hline & & acerola & amoroso & ACE AMO & REF \\
\hline \multirow{10}{*}{2} & 1 & inventar & trilho & INV TRI & GRA \\
\hline & & apoio & cativar & APO CAT & REV \\
\hline & 2 & assinatura & palha & ASS PAL & EXP \\
\hline & & anta & baleia & ANT BAL & MEN \\
\hline & 3 & analfabeto & ferrugem & ANA FER & CRI \\
\hline & & baiano & porque & BAI POR & IND \\
\hline & 4 & casa & peneira & CAS PEN & TRE \\
\hline & & serra & mercadoria & SER MER & CAM \\
\hline & 5 & avestruz & barba & AVE BAR & GAL \\
\hline & & amor & refeição & AMO REF & ACE \\
\hline \multirow{10}{*}{3} & 1 & trigo & grampo & TRI GRA & INV \\
\hline & & catar & revelar & CAT VER & $\mathrm{APO}$ \\
\hline & 2 & palito & explicar & PAL EXP & ASS \\
\hline & & balde & menta & BAL MEN & ANT \\
\hline & 3 & ferramenta & crítico & FER CRI & ANA \\
\hline & & porco & índio & POR IND & BAI \\
\hline & 4 & pente & treino & PEN TER & CAS \\
\hline & & mercado & camisa & MER CAM & SER \\
\hline & 5 & barco & galeria & BAR GAL & AVE \\
\hline & & referência & aceitar & REF ACE & AMO \\
\hline
\end{tabular}

Nota: À cada grupo eram apresentadas duas palavras de alta probabilidade e duas de baixa probabilidade dentro de cada faixa de complementação de acordo com a palavra com maior probabilidade de complementação; na recordação, cada grupo completava tríades correspondentes às palavras apresentadas (familiares), bem como a duas tríades de palavras não apresentadas (não familiares) em cada faixa. As palavras e tríades foram balanceadas entre grupos.

com a velocidade de apresentação; e c) oito palavras utilizadas para evitar a maior memorização das primeiras e últimas palavras, efeitos conhecidos como de primazia e recência, respectivamente (Capitani, Sala, Logie \& Spinnler, 1992), quatro após os exemplos e quatro ao final da lista. Assim, as palavras de cada lista de apresentação consistiam de 4 palavras que completavam tríades de cada uma das 5 faixas. Metade das palavras de cada faixa correspondia a estímulos com APC e a outra metade com BPC.

2. Lista teste (ver tabela 2): uma lista de 30 tríades foi compilada para o teste de complementação (para recordação dos estímulos), tríades essas que completavam as vinte palavras vistas (familiares) pelos sujeitos na fase de apresentação e dez palavras não vistas (utilizadas para determinar complementação ao acaso).

\section{Procedimento}

O procedimento foi o mesmo do experimento 1 no que se refere à aprovação pela Comissão de Ética Médica da Uni- versidade Federal de São Paulo, uso de sujeitos em classes e assinatura do termo de consentimento informado. As instruções foram dadas através de diapositivos. O experimento envolveu duas fases:

1. Fase de apresentação (apresentação perceptual de estímulos): consistiu na projeção de 46 palavras através de diapositivos, individualmente por $2 \mathrm{~s}$, a intervalos de $3 \mathrm{~s}$. Os sujeitos foram instruídos a ler cada palavra e marcar numa folha de resposta quantos espaços circunscritos suas letras formam (por exemplo, B tem dois espaços circunscritos, A, O e D tem um, E, G e U não têm nenhum). Essa tarefa de codificação perceptual visava diminuir a probabilidade de memorização explícita das palavras (ver Richardson-Klavehn \& Bjork, 1988; Toth, 2000). A apresentação das palavras em faixas e itens foi balanceada entre grupos experimentais (ver tabela 2). Por exemplo, para cada faixa um grupo foi exposto a duas palavras com APC que correspondiam às tríades de itens 1 e 2, duas com BPC que completavam os itens 3 e 4 e não viam as palavras correspondentes às tríades dos itens 
5 e 6. Outro grupo foi apresentado a palavras com APC referentes aos itens 3 e 4, BPC dos itens 5 e 6 e não viam palavras que completavam os itens 1 e 2 , e assim por diante. Uma tarefa de interferência (soma dos espaços circunscritos das palavras vistas) foi realizada antes da fase de teste para minimizar reverberações de palavras vistas e assim diminuir a complementação através de memória explícita.

2. Fase de teste (complementação indireta de tríades): neste teste os sujeitos deveriam completar 30 conjuntos de 3 letras (tríades) que representam inícios de palavras para formar "as primeiras palavras que lhes viessem à mente" (instrução indireta). As variáveis dependentes foram as probabilidades de complementação com estímulos de APC e BPC. Assim, obtiveram-se quatro tipos de probabilidades para cada tríade: a) probabilidades de complementação com palavras com APC quando essa palavra foi vista na fase de apresentação; b) probabilidades de complementação com palavras com APC quando nenhuma palavra que completava aquela tríade foi vista (complementação ao acaso); c) probabilidade de complementação com palavras com BPC quando essa palavra foi vista; e d) de complementação com palavras com BPC quando nenhuma palavra que completava aquela tríade foi vista (probabilidade ao acaso). A memória para um estímulo foi determinada quando a probabilidade de complementação de uma tríade com a palavra vista na fase de estudo foi maior que ao acaso.

\section{Análise estatística}

A criação do desenho experimental do presente projeto ocorreu em parceria com um grupo de profissionais do "Centro de Estatística Aplicada (CEA) da Faculdade de Matemática e Estatística da USP”, Dessa forma, a caracterização dos estímulos empregados, o número de faixas, estímulos, grupos e sujeitos a serem avaliados foram determinados de modo que a análise estatística proposta pudesse determinar o efeito de probabilidade estimada de complementação da palavra mais freqüentemente utilizada para completar uma tríade ao acaso sobre a memória para estímulos com alta e baixa probabilidade de complementação. Vale frisar que nenhuma análise estatística comumente empregada em experimentos na área de psicologia permite estudar esses efeitos.

Os três grupos de sujeitos do experimento 2 eram equiparáveis no que se refere a idade e escolaridade (testes t, $\mathrm{ps}>0,10)$ e distribuição de gêneros $\left(X^{2}, \mathrm{p}>0,10\right)$. Portanto, seus resultados foram considerados conjuntamente sem que grupo formasse um fator na análise. Para avaliar a adequação das tríades para detectar efeitos de memória, utilizou-se uma Análise de Dados Categorizados (modelo linear geral) (Agresi, 1990) com dois fatores: exposição [com 3 níveis: palavra de baixa probabilidade estimada de complementação (BPC), de alta probabilidade estimada de complementação (APC) ou nenhuma exposição] e faixa (com 5 níveis). Essa análise foi aplicada a respostas dadas com palavras de: a) baixa probabilidade estimada de complementação (BPC) e b) alta probabilidade estimada de complementação (APC). Respostas com palavras que não as com APC e BPC não foram consideradas nas análises.
Para testar a hipótese de interação entre faixa e exposição, foi utilizada a parametrização dos desvios médios e, para as hipóteses de coincidência entre perfis, utilizou-se a parametrização das médias das caselas. Os detalhes técnicos de cada parametrização estão disponíveis no site www.unifesp.br/ dpsicobio/adap/adapta.htm.

\section{Resultados}

Comparação das complementações ao acaso das amostras do experimento 1 e 2: A complementação ao acaso de palavras com BPC e APC verificada para a amostra de universitários (experimento 2) mostrou-se semelhante à determinada com estudantes de segundo grau do experimento 1, descritivamente falando: palavras com BPC foram completadas ao acaso com probabilidades de aproximadamente $5 \%$ e cresceram linearmente de 11 a $44 \%$ nas palavras de alta probabilidade estimada de complementação (APC) (ver tabelas 3 e 4 ).

Estimativas de memória para palavras familiares de baixa probabilidade estimada de complementação $(B P C)$ : não houve evidências de interação entre Exposição e Faixa ( $p=$ 0,21 ), portanto a hipótese de coincidência entre os perfis de complementação com BPC e complementação ao acaso pôde ser testada de uma única vez, já que a inexistência de interação indica que as diferenças (se existirem) são semelhantes em todas as faixas. O teste apresentou um nível descritivo bastante significativo $(p<0,001)$, indicando que a proporção de respostas dadas com BPC foi maior entre indivíduos que foram expostos a essas palavras do que em indivíduos que não viram esses estímulos e os utilizaram ao acaso como complementação. Ou seja, houve efeito de memória em todas as faixas. Também foram realizadas análises ignorando a inexistência de interação e fazendo comparações entre complementações com palavras com BPC e acaso em cada faixa. Essa análise mostrou que a superioridade de complementação com palavras com BPC quando essas são apresentadas em relação ao acaso, ou efeito de memória, de fato ocorre ( $p s<$ 0,03 ), exceto na faixa 1 (tabela 3 ).

Estimativas de memória para palavras familiares de alta probabilidade estimada de complementação (APC): neste caso houve uma indicação de interação $(p=0,090)$ entre exposição e faixa. Como o interesse era comparar a exposição de palavras com APC e sua complementação ao acaso testouse novamente a hipótese de interação considerando apenas estes 2 níveis. O resultado obtido foi $p=0,067$, indicando ainda mais a interação entre exposição e faixa, o que sugere que as possíveis diferenças nas estimativas de memória dependem de cada faixa. Pode-se observar na tabela 4 que efeitos de memória foram observados apenas nas faixas 2,3 e 4, onde a proporção de respostas com palavras com APC foram maiores para os indivíduos expostos à essas palavras do que ao acaso $(p s<0,04)$.

Os efeitos de memória para palavras com APB e BPC em cada faixa podem ser verificados na figura 1 . Resumidamente, para respostas dadas com palavras com BPC nas faixas maiores que a primeira é possível detectar memória na complementação de tríades. Ou seja, pessoas expostas a palavras com BPC completam tríades com essas palavras em maior proporção que ao acaso. Para respostas dadas com 
Tabela 3. Proporção de respostas dadas com palavras de baixa probabilidade de complementação (BPC; 4 a 7\%) comparando a complementação na presença (memória) e na ausência (complementação ao acaso) de exposição de acordo com a faixa de probabilidade de complementação.

\begin{tabular}{lcccc}
\hline \multicolumn{1}{c}{ Faixa } & Exposição Baixa & $\begin{array}{c}\text { Nenhuma Exposição } \\
\text { (Acaso) }\end{array}$ & Diferença & $\boldsymbol{p}$ \\
\hline $1(5-15 \%)$ & 0,0756 & 0,0581 & 0,0175 & 0,5170 \\
$2(16-25 \%)$ & 0,1337 & 0,0581 & 0,0756 & $0,0164 *$ \\
$3(26-35 \%)$ & 0,1453 & 0,0698 & 0,0755 & $0,0226 *$ \\
$4(36-45 \%)$ & 0,1395 & 0,0465 & 0,0930 & $0,0026 *$ \\
$5(46-55 \%)$ & 0,1570 & 0,0407 & 0,1163 & $0,0002 *$ \\
Média & 0,1302 & 0,0546 & 0,0774 & $<0,001 *$ \\
\hline
\end{tabular}

Nota: $* p s<0,03$.

Tabela 4. Proporção de respostas dadas com palavras de alta probabilidade de complementação (APC, que aumenta de faixa para faixa) comparando a complementação na presença (memória) e na ausência (complementação ao acaso) de exposição de acordo com faixa de probabilidade de complementação.

\begin{tabular}{lcccc}
\hline \multicolumn{1}{c}{ Faixa } & Exposição Alta & $\begin{array}{c}\text { Nenhuma Exposição } \\
\text { (Acaso) }\end{array}$ & Diferença & p \\
\hline $1(5-15 \%)$ & 0,1570 & 0,1163 & 0,0407 & 0,2710 \\
$2(16-25 \%)$ & 0,3430 & 0,1744 & 0,1686 & $0,0003 *$ \\
$3(26-35 \%)$ & 0,3837 & 0,1860 & 0,1977 & $<0,0001^{*}$ \\
$4(36-45 \%)$ & 0,4942 & 0,3837 & 0,1105 & $0,0378 *$ \\
$5(46-55 \%)$ & 0,5233 & 0,4419 & 0,0814 & 0,1296 \\
\hline
\end{tabular}

Nota: *ps $<0,04$

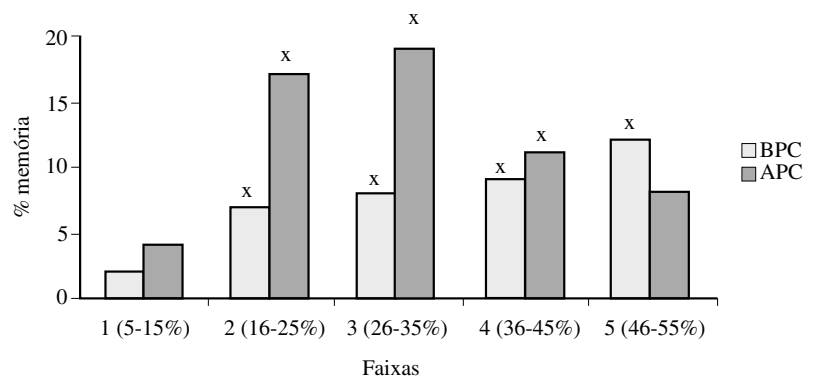

Figura 1. Estimativas de memória por faixa de probabilidade de complementação para palavras de alta (APC) e baixa (BPC) probabilidade de complementação. *complementação com palavras familiares superior à complementação ao acaso.

palavras com APC também é possível detectar memória na complementação das tríades (complementação maior que ao acaso), mas somente nas faixas 2,3 e 4 .

\section{Discussão}

A semelhança na complementação ao acaso para as amostras de estudantes universitários e de segundo grau indica que a seleção de estímulos do presente estudo foi adequada e representativa das faixas que foram criadas.

Foi demonstrado no experimento 2 que quando o teste de complementação de tríades é empregado para avaliar a memória é essencial considerar a probabilidade estimada de complementação das tríades com a palavra mais freqüentemente utilizada para completá-las ao acaso. Dependendo dessa probabilidade, não é possível verificar efeitos de memória, ou seja, a taxa de complementação de estímulos familiares, ou previamente expostos, não é distinguível da complementação ao acaso.
A ausência de interação entre efeito de exposição e faixa para palavras familiares de baixa probabilidade estimada de complementação ao acaso (BPC; 4-7\%) mostra que estas palavras, independentemente da faixa em que sua tríade seja incluída, permitem a verificação da memória, já que a prévia exposição a estas palavras eleva a complementação significativamente acima da complementação ao acaso. Entretanto, quando os contrastes são realizados faixa a faixa, nota-se que este efeito não é aplicável à faixa 1 (5-15\%) (tabela 3), tornando desaconselhável a utilização de tríades que podem ser incluídas nesta faixa para avaliar a memória.

No que se refere às palavras com alta probabilidade estimada de complementação (APC), observou-se interação de exposição e faixa indicativa de que palavras familiares com APC, às quais correspondem tríades nas faixas 2, 3 e 4 (com probabilidades de complementação entre 16-45\%), são adequadas para detectar efeitos de memória. Em contraste, a prévia exposição de palavras com APC com probabilidades entre 5 e $15 \%$ (faixa 1) e mais de $45 \%$ (faixa 5) não eleva a complementação acima da linha de base, o que implica que não é possível verificar efeitos de memória quando sujeitos são expostos a palavras com APC que começam com tríades que se encaixam nessas duas faixas.

\section{Discussão Geral}

Os resultados obtidos nos 2 experimentos aqui realizados mostram que o método utilizado internacionalmente de construir diferentes listas/versões de estímulos a serem utilizadas num mesmo estudo, através da simples equiparação da probabilidade média de complementação ao acaso de diferentes listas, não é suficiente para assegurar que ambas sejam capazes de avaliar a memória da mesma forma. Digamos, por exemplo, que duas listas sejam construídas de modo a ter, em média, $30 \%$ de probabilidade estimada de 
complementação ao acaso, mas que uma delas seja composta inteiramente de palavras que se enquadrem na faixa 3 e que exibam tendência de complementação próxima de $30 \%$ e a outra composta metade de palavras que se enquadrem na faixa 1 , com tendência de $10 \%$, e a outra metade faixa 5 , com tendência de $50 \%$. O efeito de exposição a palavras da primeira lista, ou efeito de memória, seria altamente significativo após a subtração da complementação ao acaso, ao passo que seria inexistente para a segunda lista, visto que não é possível distinguir o efeito de exposição do acaso para palavras com alta probabilidade estimada de complementação que podem ser incluídas nas faixas 1 e 5.

A título ilustrativo foram incluídos na tabela 1 a freqüência aproximada de ocorrência na língua escrita das palavras utilizadas nesse experimento (ver www.corpus.f2s.com; Sardinha, 2001). Assim como observado por Graf e Williams (1987), praticamente a metade das palavras com BPC tinham frequiências de ocorrência maiores que as de APC, mostrando mais uma vez que de fato a frequiência de uso não determina quais palavras são mais utilizadas para completar suas tríades. Desta forma, sugere-se que os seguintes critérios sejam utilizados na construção de listas de palavras para verificação de memória através do teste de complementação de tríades (ver padrões de complementação em www.unifesp.br/dpsicobio/ adap/adapta.htm):

1. Utilizar palavras às quais correspondam tríades que podem ser incluídas nas faixas 2, 3 e 4 (isto é, que tenham a palavra mais freqüentemente utilizada ao acaso com probabilidade estimada de 16 a $45 \%$ ), independentemente da tendência de complementação da palavra em si.

2. Se o objetivo do pesquisador for detectar efeitos robustos de memória em testes de complementação de fácil realização, sugere-se que utilizem palavras que se enquadrem nas faixas 2, 3 e 4 (mas especialmente faixas 2 e 3; ver figura 1) e que exibam as mais altas probabilidades de complementação dentro de sua faixa.

3. Se houver interesse em manter uma baixa probabilidade estimada de complementação para as tríades, ou seja, tornar o teste mais difícil, pode-se utilizar palavras com probabilidades de complementação baixa que exibam tríades que possam ser completadas por ao menos uma palavra com probabilidade entre $16-45 \%$ (ou seja, que se enquadrem nas faixas 2, 3, e 4). Deve-se manter em mente, contudo, que a utilização de palavras com probabilidade estimada de complementação ao acaso baixas pode inviabilizar a determinação de memória quando amostras pequenas são empregadas.

$\mathrm{O}$ fato de se ter utilizado no experimento 2 um teste indireto de complementação implica em que os critérios acima apontados para a construção de listas de palavras para verificação de memória talvez não sejam os mesmos quando instruções diretas são de interesse. Embora isso somente possa ser averiguado em estudos posteriores, acreditamos que a seleção de estímulos aqui proposta, que é muito mais rigorosa que a aplicada na literatura em geral, seja também válida para experimentos que empreguem instruções diretas para o teste de complementação.

Para os interessados em obter estimativas paralelas, nos mesmos sujeitos, de memória explícita e implícita usando o teste de complementação de tríades, sugerimos que seja empregado o paradigma que Schacter, Bowers e Booker (1989) denominaram de Critério de Recordação Intencional (Retrieval Intentionality Criterion), que envolve a verificação de diferenças estatísticas no desempenho em testes diretos e indiretos com as mesmas características salvo as instruções (para ampla discussão, diretrizes e texto em português ver Pompéia, 2000 e Pompéia \& Bueno, submetido). Outro paradigma de investigação de memória que permite quantificar a contribuição da memória explícita e implícita na realização de testes como o de complementação de tríades é o Procedimento de Dissociação de Processos (Process-Dissociation Procedure; Jacoby, 1998; Jacoby e cols., 1993), uma metodologia bastante mais complexa também amplamente discutida por Pompéia (2000). Caso haja interesse em usar testes de complementação que não envolvam palavras e sim figuras de objetos comuns, já existem testes adaptadas para o Brasil (Pompéia \& Bueno, 1996; ver também Pompéia, Miranda \& Bueno, 2001).

Por fim, apesar da semelhança entre os perfis de complementação entre estudantes universitários e de segundo grau, é importante enfatizar que as probabilidades ao acaso aqui descritas servem somente como guias para outras populações, como a de pacientes com déficits de linguagem, distúrbios neurológicos e psiquiátricos, pessoas com outros níveis de escolaridade, idades ou de outras regiões do país. Em cada caso, para a construção de testes específicos, faz-se necessária a realização de estudos-piloto sobre o perfil de complementação das populações-alvo.

\section{Referências}

Agresi, A. (1990). Categorical Data Analysis. New York, Wiley. Capitani, E.; Sala, D.D.; Logie, R.H. \& Spinnler, H. (1992). Recency, primacy and memory: reappraising and standardising the serial position curve. Cortex, 28, 315-342.

Ferreira, A.B.H. (1993). Minidicionário da língua portuguesa. $3^{\text {a }}$ edição revisada e ampliada. Rio de Janeiro, Editora Nova Fronteira.

Graf, P. \& Schacter, D.L. (1985). Implicit and explicit memory for new associations in normal and amnesic subjects. Journal of Experimental Psychology: Learning, Memory, \& Cognition, 11, 501-18.

Graf, P. \& Williams, D. (1987). Completion norms of 40 three-letter word stems. Behavioral Research Methods, Instruments \& Computers, 19, 422-445.

Jacoby, L.L. (1998). Performance in automatic influences of memory: towards a user's guide for the process dissociation procedure. Journal of Experimental Psychology: Learning, Memory, \& Cognition, 24, 3-26.

Jacoby, L.L.; Toth, J.P. \& Yonelinas, A.P. (1993). Separating conscious and unconscious influences of memory: measuring recollection. Journal of Experimental Psychology: General, 122, 139-54.

Johnson, M.K. \& Hasher, L. (1987). Human learning and memory. Annual Review of Psychology, 38, 631-668.

Magila, M.C. \& Xavier, G.F. (1999). Modelos de memória de longa duração em humanos. Psicologia, Teoria e Pesquisa, $15,37-44$.

Pompéia, S. (2000). Benzodiazepínicos e cognitição: efeitos típicos e atípicos em voluntários normais. Tese de Doutorado, De- 
partamento de Psicobiologia da Universidade Federal de São Paulo, São Paulo.

Pompéia, S. \& Bueno, O.F.A. (1998). Preliminary adaptation into Portuguese of a standerdised picture set for use in research and neuropsychological assessment. Arquivos de Neuropsiquiatria, 56, 366-374.

Pompéia, S.; Miranda, M.C. \& Bueno, O.F.A. (2001). A set of 400 pictures standardised for Portuguese: norms for name agreement, familiarity and visual complexity for children and adults. Arquivos de Neuropsiquiatria, 59, 330-337.

Richardson-Klavehn, A. \& Bjork, R.A. (1988). Measures of memory. Annual Review of Psychology, 39, 475-543.

Sardinha, T.B. (1991). The bank of Portuguese - A multi-million word monitor corpus of Brazilian Portuguese. Trabalho apresentado no 4th Corpus Linguistics Symposium, InPLA 2001.
PUC/SP, São Paulo, Brasil. (http://www.tonyberber.f2s.com/ 2000_inpla_banco_port.pdf).

Schacter, D.L.; Bowers J.S. \& Booker J. (1989). Intention, awareness and implicit memory, the retrieval intentionality criterion. Em: S. Lewandowsky, J.C. Dunn, K. Kirsner (Orgs.). Implicit memory: theoretical issues (pp. 47-64). Hillsdale, Lawrence Erlbaum Associates.

Toth, J.P.; Lindsay, S. \& Jacoby, L.L. (1992). Awareness, automaticity, and memory dissociations. Em: L.R. Squire \& N. Butters (Orgs.). The neuropsychology of memory 2a Edição. (pp. 46-57). New York, Guilford Press.

Toth, J.P. (2000). Nonconscious forms of human memory. Em: E. Tulving \& F.I.M. Craik (Orgs.). The handbook of memory (pp. 245-266). Oxford, Oxford University Press, 2000.

Recebido em 22.11.2001

Primeira decisão editorial em 15.03.2002

Versão final em 01.03.2003

Aceito em 01.04.2003 